\title{
Compact Operators for Almost Conservative and Strongly Conservative Matrices
}

\author{
S. A. Mohiuddine, ${ }^{1}$ M. Mursaleen, ${ }^{2}$ and A. Alotaibi ${ }^{1}$ \\ ${ }^{1}$ Department of Mathematics, Faculty of Science, King Abdulaziz University, P.O. Box 80203, Jeddah 21589, Saudi Arabia \\ ${ }^{2}$ Department of Mathematics, Aligarh Muslim University, Aligarh 202002, India
}

Correspondence should be addressed to M. Mursaleen; mursaleenm@gmail.com

Received 24 December 2013; Accepted 14 February 2014; Published 24 March 2014

Academic Editor: Adem Kilicman

Copyright (C) 2014 S. A. Mohiuddine et al. This is an open access article distributed under the Creative Commons Attribution License, which permits unrestricted use, distribution, and reproduction in any medium, provided the original work is properly cited.

We obtain the necessary and sufficient conditions for an almost conservative matrix to define a compact operator. We also establish some necessary and sufficient (or only sufficient) conditions for operators to be compact for matrix classes $(f, X)$, where $X=$ $c, c_{0}, l_{\infty}$. These results are achieved by applying the Hausdorff measure of noncompactness.

\section{Introduction and Preliminaries}

For some basic definitions and notations of this section we refer to $[1,2]$. Let $w$ denote the space of all complex sequences $x=\left(x_{k}\right)$, and let $\phi$ be the set of all sequences that terminate in zeros. Let $\ell_{\infty}, c$, and $c_{0}$ denote the spaces of all bounded, convergent, and null sequences, respectively. We will write $c s$ and $\ell_{1}$ for the spaces of all convergent and absolutely convergent series, respectively. Further, we will use the conventions that $e=(1,1,1, \ldots)$ and $e^{(n)}=$ $(0,0, \ldots, 1,0, \ldots)$ where 1 at the $n$th place for each $n \in \mathbb{N}=$ $\{1,2,3, \ldots\}$.

For the sequence spaces $X$ and $Y$, we write

$M(X, Y)=\left\{a=\left(a_{k}\right) \in w: a x=\left(a_{k} x_{k}\right) \in Y \forall x=\left(x_{k}\right) \in X\right\}$

which is called the multiplier space of $X$ and $Y$. The $\alpha-, \beta-$, and $\gamma$-duals of a sequence space $X$, which are respectively denoted by $X^{\alpha}, X^{\beta}$, and $X^{\gamma}$, are defined by

$$
X^{\alpha}=M\left(X, \ell_{1}\right), \quad X^{\beta}=M(X, c s), \quad X^{\gamma}=M(X, b s) .
$$

Throughout this paper, the matrices are infinite matrices of complex numbers. If $A$ is an infinite matrix with complex entries $a_{n k}(n, k \in \mathbb{N})$, then we write $A=\left(a_{n k}\right)$ instead of $A=\left(a_{n k}\right)_{n, k=1}^{\infty}$. Also, we write $A_{n}$ for the sequence in the $n$th row of $A$; that is, $A_{n}=\left(a_{n k}\right)_{k=1}^{\infty}$ for every $n \in \mathbb{N}$. In addition, if $x=\left(x_{k}\right) \in w$, then we define the A-transform of $x$ as the sequence $A x=\left(A_{n}(x)\right)_{n=1}^{\infty}$, where

$$
A_{n}(x)=\sum_{k=1}^{\infty} a_{n k} x_{k} ; \quad(n \in \mathbb{N})
$$

provided the series on the right converges for each $n \in \mathbb{N}$.

For arbitrary sequence spaces $X$ and $Y$, we write $(X, Y)$ for the class of all infinite matrices that map $X$ into $Y$. Thus $A \in(X, Y)$ if and only if $A_{n} \in X^{\beta}$ for all $n \in \mathbb{N}$ and $A x \in Y$ for all $x \in X$.

The theory of $B K$ spaces is the most powerful tool in the characterization of matrix transformations between sequence spaces.

A sequence space $X$ is called a $B K$ space if it is a Banach space with continuous coordinates $p_{n}: X \rightarrow \mathbb{C}(n \in \mathbb{N})$, where $\mathbb{C}$ denotes the complex field and $p_{n}(x)=x_{n}$ for all $x=\left(x_{k}\right) \in X$ and every $n \in \mathbb{N}$.

The sequence spaces $c_{0}, c$, and $\ell_{\infty}$ are $B K$ spaces with the usual sup norm given by $\|x\|_{\ell_{\infty}}=\sup _{k}\left|x_{k}\right|$, where the supremum is taken over all $k \in \mathbb{N}$. Also, the space $\ell_{1}$ is a $B K$ space with the usual $\ell_{1}$-norm defined by $\|x\|_{\ell_{1}}=\sum_{k=1}^{\infty}\left|x_{k}\right|$.

If $X \supset \phi$ is a $B K$ space and $a=\left(a_{k}\right) \in w$, then we write

$$
\|a\|_{X}^{*}=\sup _{x \in S_{X}}\left|\sum_{k=1}^{\infty} a_{k} x_{k}\right|
$$


provided the expression on the right exists and is finite which is the case whenever $a \in X^{\beta}$, where $S_{X}$ is the unit sphere in $X$; that is, $S_{X}=\{x \in X:\|x\|=1\}$.

A sequence $\left(b_{k}\right)_{k=0}^{\infty}$ in a linear metric space $(X, d)$ is called a Schauder basis (or briefly basis) for $X$ if for every $x \in X$ there exists a unique sequence $\left(\alpha_{k}\right)_{k=0}^{\infty}$ of scalars such that $x=$ $\sum_{k=1}^{\infty} \alpha_{k} b_{k}$; that is, $d\left(x, x^{[n]}\right) \rightarrow 0(n \rightarrow \infty)$, where $x^{[n]}=$ $\sum_{k=0}^{n} \alpha_{k} b_{k}$ is known as the $n$-section of $x$. The series $\sum_{k} \alpha_{k} b_{k}$ which has the sum $x$ is called the expansion of $x$, and $\left(\alpha_{k}\right)$ is called the sequence of coefficients of $x$ with respect to the basis $\left(b_{k}\right)$.

Let $X$ and $Y$ be Banach spaces. Then, we write $\mathscr{B}(X, Y)$ for the set of all bounded linear operators $L: X \rightarrow Y$, which is a Banach space with the operator norm given by $\|L\|=\sup _{x \in S_{X}}\|L(x)\|_{Y}$ for all $L \in \mathscr{B}(X, Y)$. A linear operator $L: X \rightarrow Y$ is said to be compact if the domain of $L$ is all of $X$ and for every bounded sequence $\left(x_{n}\right)$ in $X$, the sequence $\left(L\left(x_{n}\right)\right)$ has a subsequence which converges in $Y$. An operator $L \in \mathscr{B}(X, Y)$ is said to be of finite rank if $\operatorname{dim} R(L)<\infty$, where $R(L)$ denotes the range space of $L$. An operator of finite rank is clearly compact. Further, we write $\mathscr{C}(X, Y)$ for the class of all compact operators from $X$ to $Y$. Let us remark that every compact operator in $\mathscr{C}(X, Y)$ is bounded; that is, $\mathscr{C}(X, Y) \subset \mathscr{B}(X, Y)$. More precisely, the class $\mathscr{C}(X, Y)$ is a closed subspace of the Banach space $\mathscr{B}(X, Y)$ with the operator norm.

Finally, the following known results are fundamental for our investigation.

Lemma 1. Let $X$ denote any of the spaces $c_{0}, c$, or $\ell_{\infty}$. Then, one has $X^{\beta}=\ell_{1}$ and $\|a\|_{X}^{*}=\|a\|_{\ell_{1}}$ for all $a \in \ell_{1}$.

Lemma 2. Let $X$ and $Y$ be BK spaces. Then, one has $(X, Y) \subset$ $\mathscr{B}(X, Y)$; that is, every matrix $A \in(X, Y)$ defines an operator $L_{A} \in \mathscr{B}(X, Y)$ by $L_{A}(x)=$ Ax for all $x \in X$.

\section{The Hausdorff Measure of Noncompactness}

Most of the definitions, notations, and basic results of this section are taken from [3]. Throughout, we will write $\mathscr{M}_{X}$ for the collection of all bounded subsets of a metric space $(X, d)$. If $Q \in \mathscr{M}_{X}$, then the Hausdorff measure of noncompactness of the set $Q$, denoted by $\chi(Q)$, is defined to be the infimum of the set of all reals $\epsilon>0$ such that $Q$ can be covered by a finite number of balls of radii $<\epsilon$ and centers in $X$. This can equivalently be redefined as follows:

$$
\chi(Q)=\inf \{\epsilon>0: Q \text { has a finite } \epsilon-\text { net }\}
$$

The function $\chi: \mathscr{M}_{X} \rightarrow[0, \infty)$ is called the Hausdorff measure of noncompactness.

If $Q, Q_{1}$, and $Q_{2}$ are bounded subsets of a metric space $X$, then we have

$$
\begin{aligned}
& \chi(Q)=0 \quad \text { if and only if } Q \text { is totally bounded, } \\
& Q_{1} \subset Q_{2} \text { implies } \chi\left(Q_{1}\right) \leq \chi\left(Q_{2}\right) .
\end{aligned}
$$

Further, if $X$ is a normed space, then the function $\chi$ has some additional properties connected with the linear structure; for example,

$$
\begin{gathered}
\chi\left(Q_{1}+Q_{2}\right) \leq \chi\left(Q_{1}\right)+\chi\left(Q_{2}\right), \\
\chi(\alpha Q)=|\alpha| \chi(Q) \quad \forall \alpha \in \mathbb{C} .
\end{gathered}
$$

Let $X$ and $Y$ be Banach spaces and $\chi_{1}$ and $\chi_{2}$ be the Hausdorff measures of noncompactness on $X$ and $Y$, respectively. An operator $L: X \rightarrow Y$ is said to be $\left(\chi_{1}, \chi_{2}\right)$-bounded if $L(Q) \in$ $\mathscr{M}_{Y}$ for all $Q \in \mathscr{M}_{X}$ and there exists a constant $C \geq 0$ such that $\chi_{2}(L(Q)) \leq C \chi_{1}(Q)$ for all $Q \in \mathscr{M}_{X}$. If an operator $L$ is $\left(\chi_{1}, \chi_{2}\right)$-bounded then the number $\|L\|_{\left(\chi_{1}, \chi_{2}\right)}:=\inf \{C \geq 0$ : $\chi_{2}(L(Q)) \leq C \chi_{1}(Q)$ for all $\left.Q \in \mathscr{M}_{X}\right\}$ is called the $\left(\chi_{1}, \chi_{2}\right)$ measure of noncompactness of $L$. If $\chi_{1}=\chi_{2}=\chi$, then we write $\|L\|_{\left(\chi_{1}, \chi_{2}\right)}=\|L\|_{\chi}$.

Let $X$ and $Y$ be Banach spaces and $L \in \mathscr{B}(X, Y)$. Then, the Hausdorff measure of noncompactness of $L$, denoted by $\|L\|_{\chi}$, can be determined by

$$
\|L\|_{\chi}=\chi\left(L\left(S_{X}\right)\right)
$$

and we have that

$$
L \text { is compact if and only if }\|L\|_{\chi}=0 \text {. }
$$

Furthermore, the function $\chi$ is more applicable when $X$ is a Banach space. The most effective way in the characterization of compact operators between the Banach spaces is by applying the Hausdorff measure of noncompactness. The following result of Goldenštein et al. [4, Theorem 1] gives an estimate for the Hausdorff measure of noncompactness in Banach spaces with Schauder bases.

Lemma 3. Let $X$ be a Banach space with a Schauder basis $\left(b_{k}\right)_{k=0}^{\infty}$ and $E \in \mathscr{M}_{X}$ and $P_{n}: X \rightarrow X(n \in \mathbb{N})$ the projector onto the linear span of $\left\{b_{0}, b_{1}, \ldots, b_{n}\right\}$. Then, one has

$$
\begin{aligned}
& \frac{1}{a} \cdot \limsup _{n \rightarrow \infty}\left(\sup _{x \in \mathrm{Q}}\left\|\left(I-P_{n}\right)(x)\right\|\right) \\
& \quad \leq \chi(E) \leq \limsup _{n \rightarrow \infty}\left(\sup _{x \in Q}\left\|\left(I-P_{n}\right)(x)\right\|\right),
\end{aligned}
$$

where $a=\limsup _{n \rightarrow \infty}\left\|I-P_{n}\right\|$ and the operator $P_{r}: X \rightarrow X$, defined for each $r \in \mathbb{N}$ by $P_{r}(x)=\sum_{k=0}^{r} \phi_{k}(x) b_{k}(x \in X)$, is called the projector onto the linear span of $\left\{b_{0}, b_{1}, \ldots, b_{r}\right\}$. Besides, all operators $P_{r}$ and $I-P_{r}$ are equibounded, where $I$ denotes the identity operator on $X$.

In particular, the following result shows how to compute the Hausdorff measure of noncompactness in the spaces $c_{0}$ and $\ell_{p}(1 \leq p<\infty)$ which are $B K$-spaces with $A K$.

Lemma 4. Let $E$ be a bounded subset of the normed space $X$, where $X$ is $\ell_{p}$ for $1 \leq p<\infty$ or $c_{0}$. If $P_{n}: X \rightarrow X(n \in \mathbb{N})$ is the operator defined by $P_{n}(x)=x^{[n]}=\left(x_{0}, x_{1}, \ldots, x_{n}, 0,0, \ldots\right)$ for all $x=\left(x_{k}\right)_{k=0}^{\infty} \in X$, then one has

$$
\chi(E)=\lim _{n \rightarrow \infty}\left(\sup _{x \in Q}\left\|\left(I-P_{n}\right)(x)\right\|\right) .
$$


It is easy to see that for $E \in \mathscr{M}_{\ell_{p}}$

$$
\chi(E)=\lim _{n \rightarrow \infty}\left(\sup _{x \in Q_{k \geq n}}\left|x_{k}\right|^{p}\right) .
$$

Also, it is known that $\left(e, e^{(0)}, e^{(1)}, \ldots\right)$ is a Schauder basis for the space $c$ and every sequence $z=\left(z_{n}\right)_{n=0}^{\infty} \in c$ has a unique representation $z=\bar{z} e+\sum_{n=0}^{\infty}\left(z_{n}-\bar{z}\right) e^{(n)}$, where $\bar{z}=\lim _{n \rightarrow \infty} z_{n}$. Thus, one defines the projector $P_{r}: c \rightarrow c(r \in \mathbb{N})$, onto the linear span of $\left\{e, e^{(0)}, e^{(1)}, \ldots, e^{(r)}\right\}$, by

$$
P_{r}(z)=\bar{z} e+\sum_{n=0}^{r}\left(z_{n}-\bar{z}\right) e^{(n)} ; \quad(r \in \mathbb{N})
$$

for all $z=\left(z_{n}\right) \in c$ with $\bar{z}=\lim _{n \rightarrow \infty} z_{n}$. In this situation, one has the following.

Lemma 5. Let $Q \in \mathscr{M}_{c}$ and $P_{r}: c \rightarrow c(r \in \mathbb{N})$ be the projector onto the linear span of $\left\{e, e^{(0)}, e^{(1)}, \ldots, e^{(r)}\right\}$. Then, one has

$$
\begin{aligned}
& \frac{1}{2} \cdot \lim _{r \rightarrow \infty}\left(\sup _{x \in Q}\left\|\left(I-P_{r}\right)(x)\right\|_{\ell_{\infty}}\right) \\
& \quad \leq \chi(Q) \leq \lim _{r \rightarrow \infty}\left(\sup _{x \in Q}\left\|\left(I-P_{r}\right)(x)\right\|_{\ell_{\infty}}\right),
\end{aligned}
$$

where I is the identity operator on $c$.

\section{Almost Conservative Matrices}

A continuous linear functional $L$ on $\ell_{\infty}$ is said to be a Banach limit if it has the following properties: (i) $L(x)=0$ if $x=$ 0 , (ii) $L(e)=1$, and (iii) $L(S x)=L(x)$; where $S$ is a shift operator defined by $(S x)_{n}=x_{n+1}$.

A bounded sequence $x=\left(x_{k}\right)$ is said to be almost convergent (Lorentz [5]) to the value $l$ if all of its Banach limits coincide; that is, $L(x)=l$ for all Banach limits $L$.

Lorentz established the following characterization.

A sequence $x=\left(x_{k}\right)$ is almost convergent to the number $l$ if and only if $t_{p n}(x) \rightarrow l$ as $p \rightarrow \infty$ uniformly in $n$, where

$$
t_{p n}(x)=\frac{1}{p+1} \sum_{m=0}^{p} x_{m+n} ; \quad(p, n \in \mathbb{N}) .
$$

The number $l$ is called the generalized limit of $x$, and we write $l=f-\lim x$. We denote the set of all almost convergent sequences by $f$; that is,

$$
f=\left\{x \in \ell_{\infty}: \lim _{p \rightarrow \infty} t_{p n}(x)=L \text { uniformly in } n\right\} .
$$

Remark 6. Note that $c \subset f \subset \ell_{\infty}$ and each inclusion is proper.

Remark 7. Since $c \subset f \subset \ell_{\infty}$, we have $\ell_{1}=\ell_{\infty}^{\beta} \subset f^{\beta} \subset c^{\beta}=\ell_{1}$ and hence $f^{\beta}=\ell_{1}$. Therefore, it is natural by (4) and Lemma 1 that $\|a\|_{f}^{*}=\|a\|_{\ell_{1}}$ for all $a \in \ell_{1}$.
Remark 8 (see [6]). $f$ is a BK-space with $\|\cdot\|_{\infty}$.

Remark 9 (see [6]). $f$ is a nonseparable closed subspace of $\left(\ell_{\infty},\|\cdot\|_{\infty}\right)$.

Using the idea of almost convergence, King [7] defined and characterized the almost conservative and almost regular matrices.

An infinite matrix $A=\left(a_{n k}\right)_{n, k=1}^{\infty}$ is said to be almost conservative if $A x \in f$ for all $x \in c$, and we denote it by $A \in(c, f)$. If in addition $f-\lim A x=\lim x$, then $A$ is called almost regular.

Remark 10 (see [7, Theorem 1]). A matrix $A=\left(a_{n k}\right)_{n, k=1}^{\infty}$ is almost conservative if and only if

(i) $\|A\|=\sup _{n}\left(\sum_{k=1}^{\infty}\left|a_{n k}\right|\right)<\infty$,

(ii) $a_{(k)}=\left(a_{n k}\right)_{n=1}^{\infty} \in f$ for each $k \in \mathbb{N}$,

(iii) $a=\left(\sum_{k=1}^{\infty} a_{n k}\right)_{n=1}^{\infty} \in f$.

Now, we prove the following.

Theorem 11. Let $A=\left(a_{n k}\right)$ be an almost conservative matrix. Then, one has

$$
\begin{gathered}
0 \leq\left\|L_{A}\right\|_{\chi} \leq \limsup _{n \rightarrow \infty}\left(\sum_{k=1}^{\infty}\left|a_{n k}\right|\right), \\
L_{A} \text { is compact if } \lim _{n \rightarrow \infty}\left(\sum_{k=1}^{\infty}\left|a_{n k}\right|\right)=0 .
\end{gathered}
$$

Proof. Let us remark that the expression on the right of (17) exists and is finite by Remark 10(i). We write $S=S_{c}$, for short. Since $A \in(c, f)$, we have by Lemma 2 that $L_{A}(S)=A S \in \mathscr{M}_{f}$. Thus, we obtain by (8) that

$$
\left\|L_{A}\right\|_{\chi}=\chi(A S) .
$$

We define the operators $P_{r}: f \rightarrow f(r \in \mathbb{N})$ by $P_{r}(x)=$ $\left(x_{1}, x_{2}, \ldots, x_{r}, 0,0, \ldots\right)$ for all $x=\left(x_{n}\right)_{n=1}^{\infty} \in f$. Then, we have

$$
A S \subset P_{r}(A S)+\left(I-P_{r}\right)(A S) ; \quad(r \in \mathbb{N}),
$$

where $I$ is the identity operator on $f$. Thus, it follows by the elementary properties of the function $\chi$ that

$$
\begin{aligned}
0 \leq \chi(A S) & \leq \chi\left(P_{r}(A S)\right)+\chi\left(\left(I-P_{r}\right)(A S)\right) \\
& =\chi\left(\left(I-P_{r}\right)(A S)\right) \\
& \leq \sup _{x \in S}\left\|\left(I-P_{r}\right)(A x)\right\|_{\ell_{\infty}}
\end{aligned}
$$

for all $r \in \mathbb{N}$. Further, we have for every $r \in \mathbb{N}$ that $\left\|\left(I-P_{r}\right)(A x)\right\|_{\ell_{\infty}}=\sup _{n>r}\left|A_{n}(x)\right|$ for all $x \in c$. Therefore, by using (3), (4), and Lemma 2 , we derive that

$$
\sup _{x \in S}\left\|\left(I-P_{r}\right)(A x)\right\|_{\ell_{\infty}}=\sup _{n>r}\left\|A_{n}\right\|_{c}^{*}=\sup _{n>r}\left\|A_{n}\right\|_{\ell_{1}} ; \quad(r \in \mathbb{N}) .
$$


Thus, we obtain that

$$
0 \leq \chi(A S) \leq \sup _{n>r}\left\|A_{n}\right\|_{\ell_{1}} ; \quad(r \in \mathbb{N})
$$

and hence

$$
\begin{aligned}
0 \leq \chi(A S) & \leq \lim _{r \rightarrow \infty}\left(\sup _{n>r}\left\|A_{n}\right\|_{\ell_{1}}\right) \\
& =\limsup _{n \rightarrow \infty}\left\|A_{n}\right\|_{\ell_{1}} .
\end{aligned}
$$

This and (19) yield (17). Finally, we get (18) from (9) and (17).

This completes the proof.

It is worth mentioning that the condition in (18) is only a sufficient condition for the operator $L_{A}$ to be compact, where $A$ is an almost conservative matrix. More precisely, the following example will show that it is possible for $L_{A}$ to be compact while $\lim _{n \rightarrow \infty}\left(\sum_{k=1}^{\infty}\left|a_{n k}\right|\right) \neq 0$. Hence, in general, we have just "if" in (18) of Theorem 11.

Example 12. Define the matrix $A=\left(a_{n k}\right)$ by $a_{n 1}=1$ and $a_{n k}=0$ for $k>1(n \in \mathbb{N})$. Then, we have $A x=x_{1} e \in f$ for all $x=\left(x_{k}\right)_{k=1}^{\infty} \in c$ and hence $A \in(c, f)$; that is, $A$ is almost conservative. Also, it is obvious that $L_{A}$ is of finite rank and so $L_{A}$ is compact. On the other hand, we have $A_{n}=e^{(1)}$ and hence $\left\|A_{n}\right\|_{\ell_{1}}=1$ for all $n \in \mathbb{N}$. This implies that $\lim _{n \rightarrow \infty}\left\|A_{n}\right\|_{\ell_{1}}=1$.

\section{Compact Operators for Strongly Conservative Matrices}

An infinite matrix $A=\left(a_{n k}\right)_{n, k=1}^{\infty}$ is said to be strongly conservative if $A x \in c$ for all $x \in f$, and we denote it by $A \in(f, c)$. If in addition $f-\lim x=\lim A x$, then $A$ is called strongly regular (cf. [5]).

In this final section, we establish some necessary and sufficient (or only sufficient) conditions for operators to be compact for matrix classes $(f, X)$, where $X=c, c_{0}, \ell_{\infty}$.

We may begin with the following lemmas which will be needed in the sequel.

Lemma 13. If the matrix $A$ is in any of the classes $\left(f, c_{0}\right),(f, c)$, or $\left(f, \ell_{\infty}\right)$, then

$$
\sup _{n}\left(\sum_{k=1}^{\infty}\left|a_{n k}\right|\right)<\infty .
$$

Proof. This can be seen from the class $(f, c)$ characterized by Lorentz [5] and by using the fact that $\left(f, c_{0}\right) \subset\left(c, c_{0}\right),(f, c) \subset$ $(c, c)$, and $\left(f, \ell_{\infty}\right) \subset\left(c, \ell_{\infty}\right)$.

This completes the proof of the theorem.

Lemma 14. If $A \in(f, c)$, then one has

$$
\begin{gathered}
\alpha_{k}=\lim _{n \rightarrow \infty} a_{n k} \text { exists for every } k \in \mathbb{N}, \\
\alpha=\left(\alpha_{k}\right)_{k=1}^{\infty} \in \ell_{1} \\
\sup _{n}\left(\sum_{k=1}^{\infty}\left|a_{n k}-\alpha_{k}\right|\right)<\infty
\end{gathered}
$$

Proof. It is trivial that (26) holds, since $e^{(k)} \in f$ for all $k \in \mathbb{N}$. Further, by combining (26) and Lemma 13, we have for every $m \in \mathbb{N}$ that $\sum_{k=1}^{m}\left|\alpha_{k}\right| \leq \sup _{n}\left(\sum_{k=1}^{\infty}\left|a_{n k}\right|\right)<\infty$ which implies that (27) holds. Finally, it follows by (27) and Lemma 13 that (28) holds.

This completes the proof of the theorem.

Now, we prove the following result on the Hausdorff measure of noncompactness.

Theorem 15. Let $A=\left(a_{n k}\right)$ be an infinite matrix. Then, one has the following.

(i) If $A \in\left(f, c_{0}\right)$, then

$$
\left\|L_{A}\right\|_{\chi}=\limsup _{n \rightarrow \infty}\left(\sum_{k=1}^{\infty}\left|a_{n k}\right|\right) .
$$

(ii) If $A \in(f, c)$, then

$$
\begin{aligned}
& \frac{1}{2} \cdot \limsup _{n \rightarrow \infty}\left(\sum_{k=1}^{\infty}\left|a_{n k}-\alpha_{k}\right|\right) \\
& \leq\left\|L_{A}\right\|_{\chi} \leq \limsup _{n \rightarrow \infty}\left(\sum_{k=1}^{\infty}\left|a_{n k}-\alpha_{k}\right|\right),
\end{aligned}
$$

where $\alpha_{k}=\lim _{n \rightarrow \infty} a_{n k}$ for all $k \in \mathbb{N}$.

(iii) If $A \in\left(f, \ell_{\infty}\right)$, then

$$
0 \leq\left\|L_{A}\right\|_{\chi} \leq \limsup _{n \rightarrow \infty}\left(\sum_{k=1}^{\infty}\left|a_{n k}\right|\right) .
$$

Proof. Let us remark that the expressions in (29), (30), and (31) exist by Lemmas 13 and 14.

We write $S=S_{f}$. Then, we obtain by (8) and Lemma 2 that

$$
\left\|L_{A}\right\|_{\chi}=\chi(A S)
$$

For (i), we have $A S \in \mathscr{M}_{c_{0}}$. Thus, it follows by applying Lemma 3 that

$$
\chi(A S)=\lim _{r \rightarrow \infty}\left(\sup _{x \in S}\left\|\left(I-P_{r}\right)(A x)\right\|_{\ell_{\infty}}\right),
$$

where $P_{r}: c_{0} \rightarrow c_{0}(r \in \mathbb{N})$ is the operator defined by $P_{r}(x)=x^{[r]}$ for all $x \in c_{0}$. This yields that $\left\|\left(I-P_{r}\right)(A x)\right\|_{\ell_{\infty}}=$ $\sup _{n>r}\left|A_{n}(x)\right|$ for all $x \in f$ and every $r \in \mathbb{N}$. Therefore, by using (3), (4), and Remark 7, we have for every $r \in \mathbb{N}$ that

$$
\sup _{x \in S}\left\|\left(I-P_{r}\right)(A x)\right\|_{\ell_{\infty}}=\sup _{n>r}\left\|A_{n}\right\|_{f}^{*}=\sup _{n>r}\left\|A_{n}\right\|_{\ell_{1}} .
$$

This and (33) imply that

$$
\chi(A S)=\lim _{r \rightarrow \infty}\left(\sup _{n>r}\left\|A_{n}\right\|_{\ell_{1}}\right)=\limsup _{n \rightarrow \infty}\left\|A_{n}\right\|_{\ell_{1}} .
$$

Hence, we get (29) by (32). 
To prove (ii), we have $A S \in \mathscr{M}_{c}$. Thus, we are going to apply Lemma 4 to get an estimate for the value of $\chi(A S)$ in (32). For this, let $P_{r}: c \rightarrow c(r \in \mathbb{N})$ be the projectors defined by (13). Then, we have for every $r \in \mathbb{N}_{0}$ that $\left(I-P_{r}\right)(z)=$ $\sum_{n=r+1}^{\infty}\left(z_{n}-\bar{z}\right) e^{(n)}$ and hence

$$
\left\|\left(I-P_{r}\right)(z)\right\|_{\ell_{\infty}}=\sup _{n>r}\left|z_{n}-\bar{z}\right|
$$

for all $z=\left(z_{n}\right) \in c$ and every $r \in \mathbb{N}_{0}$, where $\bar{z}=\lim _{n \rightarrow \infty} z_{n}$ and $I$ is the identity operator on $c$.

Now, by using (32), we obtain by applying Lemma 5 that

$$
\begin{aligned}
& \frac{1}{2} \cdot \lim _{r \rightarrow \infty}\left(\sup _{x \in S}\left\|\left(I-P_{r}\right)(A x)\right\|_{\ell_{\infty}}\right) \\
& \quad \leq\left\|L_{A}\right\|_{\chi} \leq \lim _{r \rightarrow \infty}\left(\sup _{x \in S}\left\|\left(I-P_{r}\right)(A x)\right\|_{\ell_{\infty}}\right) .
\end{aligned}
$$

Further, since $A \in(f, c)$, we have by combining Lemmas 13 and 14 that $\alpha=\left(\alpha_{k}\right) \in \ell_{1}$ and $\lim _{n \rightarrow \infty} A_{n}(x)=\sum_{k=1}^{\infty} \alpha_{k} x_{k}$ for all $x=\left(x_{k}\right) \in f$. Consequently, we derive from (36) that

$$
\begin{aligned}
\left\|\left(I-P_{r}\right)(A x)\right\|_{\ell_{\infty}} & =\sup _{n>r}\left|A_{n}(x)-\sum_{k=1}^{\infty} \alpha_{k} x_{k}\right| \\
& =\sup _{n>r}\left|\sum_{k=1}^{\infty}\left(a_{n k}-\alpha_{k}\right) x_{k}\right|
\end{aligned}
$$

for all $x=\left(x_{k}\right) \in f$ and every $r \in \mathbb{N}_{0}$. Therefore, it follows by (4) that

$$
\begin{aligned}
\sup _{x \in S}\left\|\left(I-P_{r}\right)(A x)\right\|_{\ell_{\infty}} & =\sup _{n>r}\left\|A_{n}-\alpha\right\|_{f}^{*} \\
& =\sup _{n>r}\left\|A_{n}-\alpha\right\|_{\ell_{1}} ; \quad\left(r \in \mathbb{N}_{0}\right) .
\end{aligned}
$$

Hence, from (37) we get (30).

For (iii), we have $A S \in \mathscr{M}_{\ell_{\infty}}$. Thus, we define $P_{r}: \ell_{\infty} \rightarrow$ $\ell_{\infty}(r \in \mathbb{N})$ by $P_{r}(x)=\left(x_{1}, x_{2}, \ldots, x_{r}, 0,0, \ldots\right)$ for all $x=$ $\left(x_{k}\right) \in \ell_{\infty}$. Then, the proof can be achieved similarly as the proof of Theorem 11.

This completes the proof of the theorem.

Finally, we conclude our work by the following corollary.

Corollary 16. Let $A=\left(a_{n k}\right)$ be an infinite matrix. Then, one has the following.

(i) If $A \in\left(f, c_{0}\right)$, then

$L_{A}$ is compact if and only if $\lim _{n \rightarrow \infty}\left(\sum_{k=1}^{\infty}\left|a_{n k}\right|\right)=0$.

(ii) If $A \in(f, c)$, then

$L_{A}$ is compact if and only if $\lim _{n \rightarrow \infty}\left(\sum_{k=1}^{\infty}\left|a_{n k}-\alpha_{k}\right|\right)=0$,

where $\alpha_{k}=\lim _{n \rightarrow \infty} a_{n k}$ for all $k \in \mathbb{N}$. (iii) If $A \in\left(f, \ell_{\infty}\right)$, then

$$
L_{A} \text { is compact if } \lim _{n \rightarrow \infty}\left(\sum_{k=1}^{\infty}\left|a_{n k}\right|\right)=0 .
$$

Proof. This result follows from Theorem 15 by using (9).

Remark 17. As in our Example 12, it can be shown similarly that the equivalence in (42) of Corollary 16 does not hold.

Remark 18. Since matrix mappings between BK spaces define bounded linear operators between these spaces which are Banach spaces, it is natural to use the Hausdorff measure of noncompactness to obtain necessary and sufficient conditions for matrix operators between BK spaces to be compact operators. This technique has recently been used by several authors in many research papers (cf. [8-14]). Further the technique of measures of noncompactness has also been used in solving the infinite system of differential equations in some sequence spaces (see [15-17]).

\section{Conflict of Interests}

The authors declare that there is no conflict of interests regarding the publication of this paper.

\section{Acknowledgment}

The authors gratefully acknowledge the financial support from King Abdulaziz University, Jeddah, Saudi Arabia.

\section{References}

[1] F. Başar, Summability Theory and Its Applications, E-books, Monographs, Bentham Science, Istanbul, Turkey, 2011.

[2] A. Wilansky, Summability through Functional Analysis, vol. 85 of North-Holland Mathematics Studies, Elsevier Science, New York, NY, USA, 1984.

[3] E. Malkowsky and V. Rakočević, "An introduction into the theory of sequence spaces and measures of noncompactness," Zbornik Radova, vol. 9, no. 17, pp. 143-234, 2000.

[4] L. S. Goldenštein, I. T. Gohberg, and A. S. Markus, "Investigations of some properties of bounded linear operators with their q-norms," Ucenie Zapiski, Kishinevskii, vol. 29, pp. 29-36, 1957.

[5] G. G. Lorentz, "A contribution to the theory of divergent sequences," Acta Mathematica, vol. 80, no. 1, pp. 167-190, 1960.

[6] F. Başar and M. Kirisci, "Almost convergence and generalized difference matrix," Computers \& Mathematics with Applications, vol. 61, no. 3, pp. 602-611.

[7] J. P. King, "Almost summable sequences," Proceedings of the American Mathematical Society, vol. 17, pp. 1219-1225, 1966.

[8] B. de Malafosse, E. Malkowsky, and V. Rakočević, "Measure of noncompactness of operators and matrices on the spaces $c$ and $c_{0}$," International Journal of Mathematics and Mathematical Sciences, vol. 2006, Article ID 46930, 5 pages, 2006.

[9] M. Mursaleen and A. K. Noman, "Compactness by the Hausdorff measure of noncompactness," Nonlinear Analysis: Theory, Methods and Applications, vol. 73, no. 8, pp. 2541-2557, 2010. 
[10] F. Başar and E. Malkowsky, "The characterization of compact operators on spaces of strongly summable and bounded sequences," Applied Mathematics and Computation, vol. 217, no. 12, pp. 5199-5207, 2011.

[11] M. Başarir and E. E. Kara, "On compact operators on the Riesz $B^{m}$-difference sequence space," Iranian Journal of Science and Technology, Transaction A, vol. 35, no. 4, pp. 279-285, 2011.

[12] E. E. Kara and M. Basarir, "On compact operators and some Euler $B(m)$-difference sequence spaces," Journal of Mathematical Analysis and Applications, vol. 379, no. 2, pp. 499-511, 2011.

[13] M. Başarir and E. E. Kara, "On the $B$-difference sequence space derived by generalized weighted mean and compact operators," Journal of Mathematical Analysis and Applications, vol. 391, no. 1, pp. 67-81, 2012.

[14] S. A. Mohiuddine, M. Mursaleen, and A. Alotaibi, "The Hausdorff measure of noncompactness for some matrix operators," Nonlinear Analysis, vol. 92, pp. 119-129, 2013.

[15] J. Banaś and M. Lecko, "Solvability of infinite systems of differential equations in Banach sequence spaces," Journal of Computational and Applied Mathematics, vol. 137, no. 2, pp. 363375, 2001.

[16] M. Mursaleen and S. A. Mohiuddine, "Applications of measures of noncompactness to the infinite system of differential equations in $\ell_{p}$ spaces," Nonlinear Analysis: Theory, Methods and Applications, vol. 75, no. 4, pp. 2111-2115, 2012.

[17] M. Mursaleen, "Application of measure of noncompactness to infinite systems of differential equations," Canadian Mathematical Bulletin, vol. 56, pp. 388-394, 2013. 


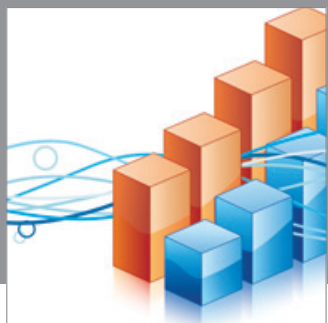

Advances in

Operations Research

mansans

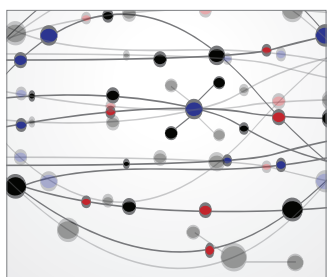

The Scientific World Journal
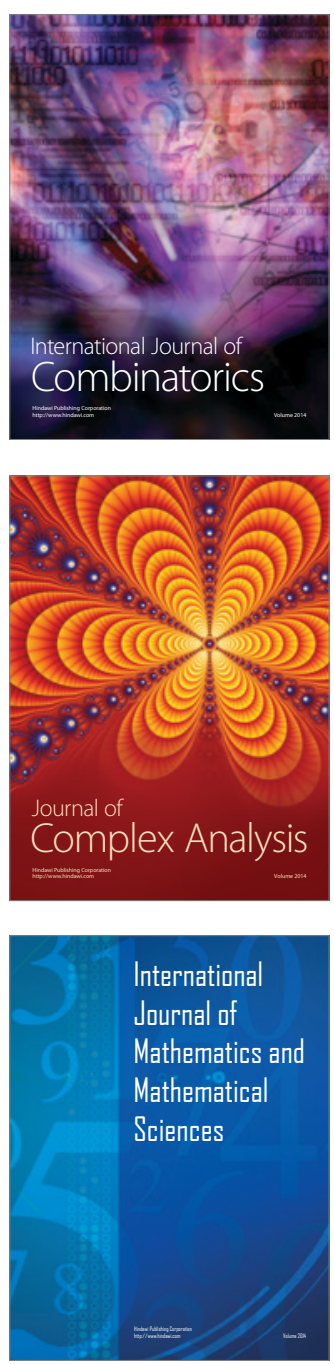
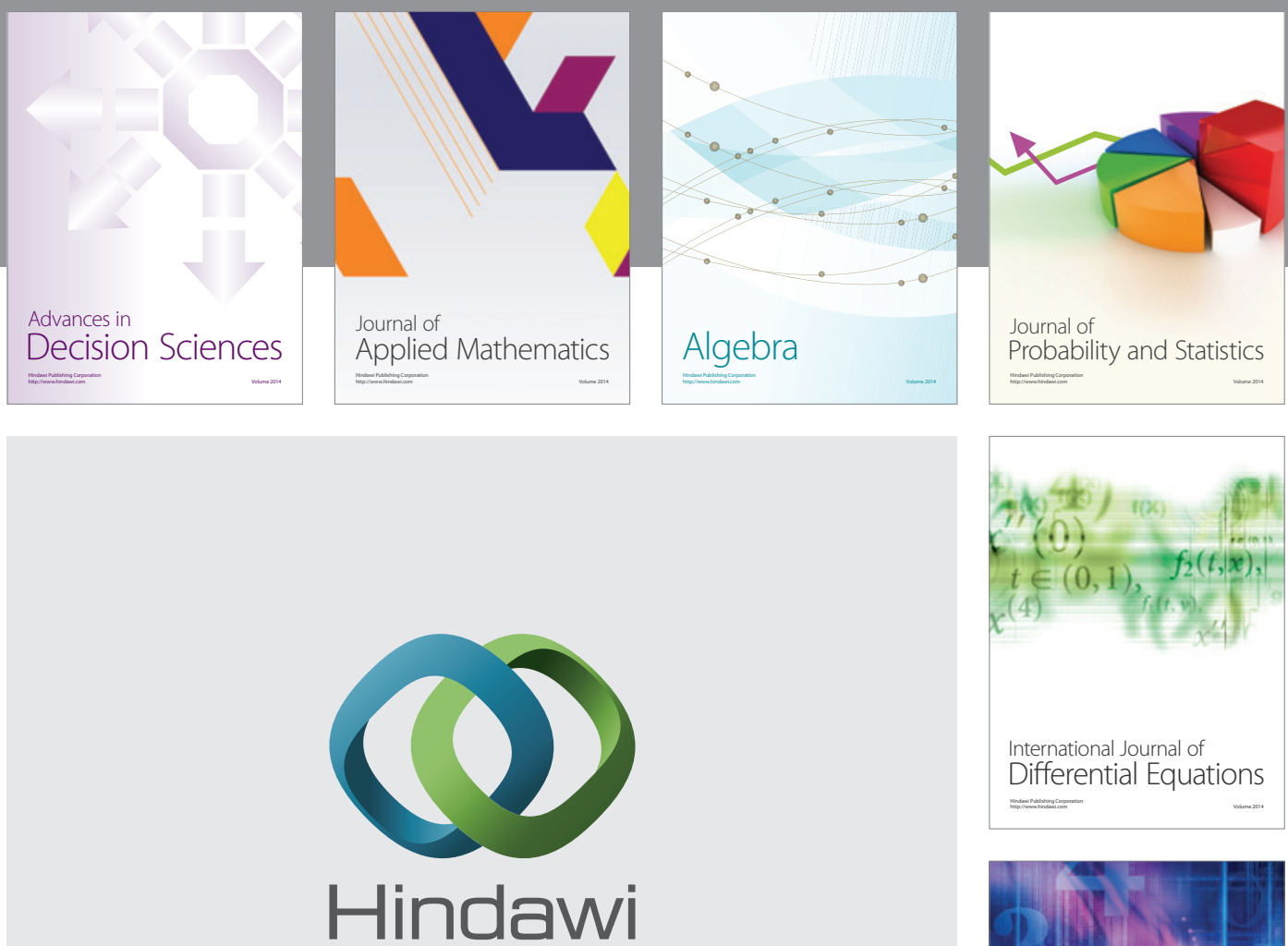

Submit your manuscripts at http://www.hindawi.com
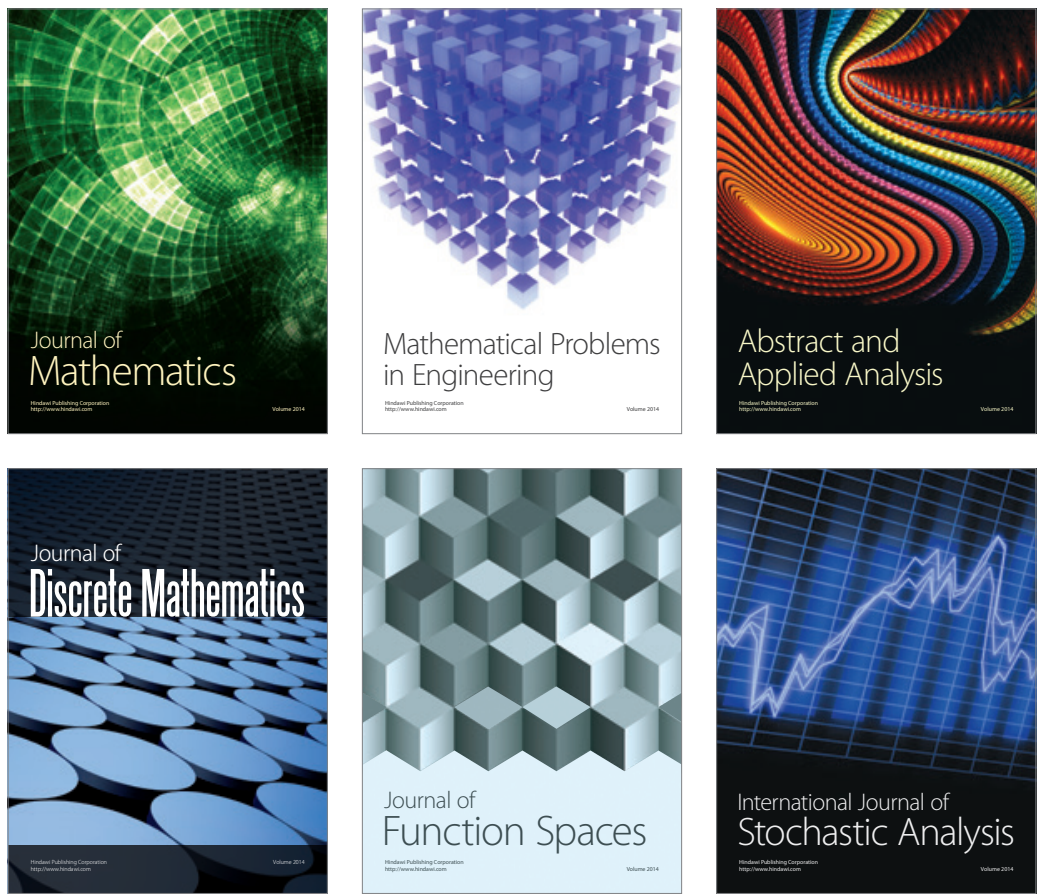

Journal of

Function Spaces

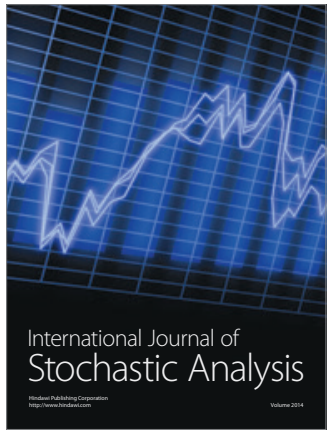

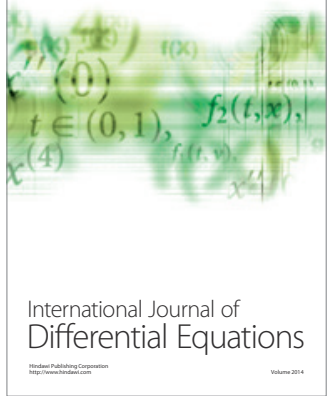
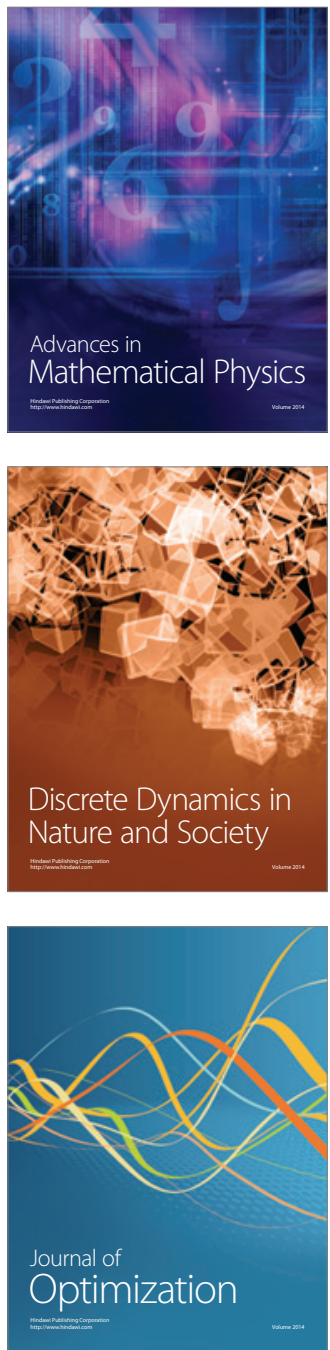